(1)rininal Artirlex.

\section{DISEASES OF THE MIDDLE EAR AND MASTOID CELLS, BASED UPON A STUDY OF 454 AUTOPSIES AND 2232 CASES OF DIPHTHERIA, SCARLET FEVER, AND MEASLES.*}

BY C. R. C. BORDEN, M.D., BOSTON.

SINCE the opening of the contagious department of the Boston City Hospital in 1896, autopsy records of 454 cases have been collected. Nearly all of them were children, who had died from scarlet fever, diphtheria, measles, or from complications arising from these diseases. The records were written by skilled pathologists who performed the autopsies, and each record is as complete, carefully written, and accurate as it is possible to make them. In 202 of the autopsies the head was not opened, owing to the objection of parents and friends. The remaining 252 state clearly the condition of the middle ear or mastoid, normal or abnormal, as the particular case may be.

In order that we may better compare the post mortem with the clinical cases, I will briefly quote the few statistics available. Dr. McCollom reports $18 \%$ of middle ear complications in scarlet fever and $24 \%$ in measles. Downie of Glasgow $12 \%$ in scarlet fever and $26 \%$ in measles. Caiger $11 \%$ in scarlet fever; Burckhart and Finlayson $10 \%$ and Alderton $11 \%$ in scarlet fever and but $15 \%$ in measles.

None of the above writers have mentioned the aural complications in diphtheria. The only statistics I know of in this disease are a small number of cases observed and reported by the writer in a previous paper, viz. 1.4\%. To further compare statistics in the same institution, I have collected a series of 2232 cases of scarlet fever, measles and diphtheria. Of this number, 746 cases of searlet fever showed $11 \%$ of aural complications; 456 cases of measles $28 \%$; and 952 cases of diphtheria $2.9 \%$. Sixtyeight cases of mixed infection were the highest in the percentage of all middle ear complications, viz. $44 \%$.

Such statistics will vary considerably in different epidemics with the season of the year, the institution in which they occur and the community from which the patients have come. Many of our children are brought to us from large charitable institutions and these little patients unfortunately constitute a considerable number of our seriously sick or fatal cases. On the other hand, the contagious hospitals of Brookline and Somerville, suburbs of Boston, show a marked decrease in the number of aural complications; due to the fact that the vitality of their patients is greater upon entering the

* Read before the Ninth International Otological Congress, at Boston, August, 1912. hospital. This fact should be clearly borne in mind in consideration of the cases to be reported in this paper, in as much as the majority of our patients come from very poor surroundings, and consequently have a relatively low degree of bodily resistance.

From the statistics gathered thus far in clinical cases, we would naturally suppose complications of the middle ear and mastoid would follow the same relative frequency in fatal cases. The frequency is the same, but the percentage is far greater. In diphtheria, instead of $2.9 \%$ it is $82 \%$. In scarlet fever it is $94 \%$ and in measles $100 \%$ of middle ear or mastoid involvement.

Again, we would naturally expect mastoiditis to follow the general course of the middle ear. As a matter of fact, however, the order is exactly reversed in the fatal cases. We find diphtheria least in the clinical, to be highest in the fatal cases in the number of mastoids. Thirty-one per cent. of fatal diphtheria cases showed mastoid involvement, $26 \%$ of the scarlet fever cases and but $14 \%$ of the measles cases had mastoiditis. This is a significant fact and is, I believe, the most important point in the entire subject.

Bilateral otitis media is far more common than unilateral in all the fatal cases, as is also bilateral mastoiditis in diphtheria and scarlet fever. In measles, but one case of mastoiditis occurred in the series of 7 fatal cases, and that one was unilateral.

Of the 59 cases of mastoiditis, 33 of them were bilateral, and in every case, conditions were ideal to create the severe allied complications which so often occur with undetected and untreated mastoiditis. Yet, the autopsy records show but one case of an infected jugular vein; but four cases of septic meningitis; and not a single brain abscess. This is made even more remarkable by the fact, that at least $80 \%$ of the mastoiditis cases showed edema and congestion of the brain. Edema of the brain was not. confined to the mastoiditis cases, however, as it occurred in a large number of the infected middle ears without mastoid involvement and in a few cases where neither middle ear nor mastoid were infected.

The appearance of the middle ear and mastoid cells, at the time of the autopsy, present a sharp contrast between scarlet fever, measles and diphtheria when these special organs are diseased. In scarlet fever and measles the fluid found therein is almost invariably described as yellow, white or creamy pus. In diphtheria, record after record refers to it as small in amount, thick, tenacious, gummy gelatinous, semi-solid, etc. The color is also peculiar in this disease, and is described as green, yellowish green, brownish green and other colors bordering on this hue. Involvement of the mastoid cells ranges from acute congestion to absolute destruction.

Of the 59 cases of mastoiditis but 6 or 8 of them were recognized during life, and of that 
number, operation was performed on but one side when both sides were diseased. I beg to state, however, that the 59 cases of mastoiditis covered a period of nearly 15 years and that our mastoid operations upon diagnosed cases show a mortality of only 2 or $3 \%$ in uncomplicated cases.

In four cases a fistulous tract was found, leading directly from the middle ear into the cranial cavity, but in only one of these cases was septic meningitis present.

Doctors Councilman, Mallory and Pearce, in an exhaustive article on diphtheria, report the cultures made in 86 of these same autopsies as follows: In 59 cases: staphylococcus was found in 7 cases; pneumococcus in 5; staphylococcus albus in 3 ; streptococcus pyogenes in 4 , and streptococcus aureas in 1 case. In a series of 27 cases; the diphtheria bacillus was found in 12 cases; streptococcus in 14; staphylococcus pyogenes in 5 ; bacillus pyocyannus in 2 cases. Generally two or more of the bacilli occurred together and, as a rule, the diphtheria bacillus occurred with the streptococcus, the staphylococcus or with both. Few pure cultures were present of any kind.

An examination of the nasal sinuses in 52 cases of diphtheria are reported in the same article. In 33 eases, inflammatory changes were present in both antra 19 ; both antra, sphenoid and ethmoid 2 ; one antrum only 7 ; sphenoid only 2 ; and in the sphenoid and ethmoid 1. In 59 of the autopsy cases, 21 acute abscess cavities were found in different parts of the body.

The question will naturally arise in your mind, why so many of the otitis media cases and most of the mastoiditis were unsuspected during the illness of the patient. It is not because the resident physicians in the hospital were negligent or careless. Whenever symptoms arose pointing to the middle ear, careful examination was made of those organs. But diagnosis of such conditions is, in many cases, extremely difficult to make. A careful daily routine aural examination of from two to five hundred patients is impossible, as physical examination of the other organs must be made at the same time. It is entirely possible to have, not only otitis media present and active without diagnostic symptoms; but mastoiditis as well. Such being the case, the attending physician cannot be criticised for failing to diagnose many aural complications.

In measles, middle ear and mastoid symptoms occur during the height of the active process, and adults are particularly liable to mastoiditis. In scarlet fever, middle ear involvement comes at any time during the course of the disease, and is by no means limited to the active stage. Adults are not especially liable to mastoiditis in scarlet fever. In diphtheria, otitis media or mastoiditis are not as active as in scarlet fever or measles, and the diagnosis is far more diffcult to make for this reason.

Middle ear complications are not only of ex- treme importance on their own account, but because of their effect upon other complications present. When they occur with inflammations in other organs, such effections are invariably exaggerated. Thus, with active inflammation in the heart, lungs or joints, infection of the middle ear or mastoid cells, causes the symptoms in those organs to become more active and dangerous to the life of the patient. On the other hand, prompt and efficient treatment of the aural inflammation, usually causes marked relief in the inflammatory process of the other organs.

Next to bony tissue itself, bony cavities, lined with mucous membrane are among the lowest of all the tissues of the human body, in their power to cope with bacterial invasion. Because of its relatively small blood supply, the antibodies cannot reach such cavities with sufficient freedom to strongly exert their anti-bacterial properties. In both the middle ear and mastoid cells, we have not only bony cavities lined with mucous membrane, but anatomical structures particularly susceptible to infection from the nose or throat, because of their location. In case of infection, if the Eustachian tube becomes sufficiently swollen to close its lumen, the middle ear and mastoid cells become closed cavities, and we have at once a condition identical with an abscess cavity in any part of the body. The passing of bacteria from an infected middle ear into the circulating blood stream produces symptoms too well known to require further mention.

So long as the phagocytes and the anti-bodies have sufficient power to overcome the bacterial invasion, no systemic symptoms occur. This is doubtless the reason why infected middle ears occasionally smoulder for weeks before showing active symptoms.

A few years ago, attention was called to the tonsil as an avenue by which bacteria from the throat and mouth could find its way into the general circulation. The discussion pro and con of this theory lasted several years and gradually it became an accepted fact. In establishing the theory, eminent writers proved conclusively, that such effections as endocarditis, pleuritis, septic synovitis, adenitis, etc., often derived their infection primarily from a diseased tonsil. In such cases, thorough removal of the infected organ was quickly followed by permanent relief in the inflamed condition. The results of such operations were so uniformly beneficial, that they have become one of the most common procedures in our daily practices.

Up to this time we have looked upon the middle ear and mastoid cells as structures particularly susceptible to infection in the contagious diseases. When such infection occurred, however, we feared only one of two things; if moderately severe, discomfort to the patient from the pain and a strong possibility of deranged hearing later; if very severe, mastoiditis. Should the process extend still further, brain 
abscess or jugular thrombosis, were the dreaded consequences to be feared. So far as I know, the middle ear or mastoid cells have never been suspected of being the primary foci of infection in the many and serious complications which so commonly occur in the contagious diseases. Yet the position of these bony cavities is identical in every way with the tonsil as an avenue of entrance for bacteria into the circulation. Anatomically their structure is ideal to produce inflammation, and their location is such as to be closely related to the specifically inflamed tissues of these particular diseases. Previously we have supposed the percentage of aural involvement to be relatively small, but the study of the fatal cases, proves them to be alarmingly high. As practically all the fatal cases showed complications in the heart, lung, kidneys, etc., as well as in the middle ear or mastoid cells, may we not suspect the two to go hand in hand, to a very great extent? Indeed we may go still further and assume one to depend upon the other.

Many of the clinical records show a sudden rise in temperature followed by all manner of complications in various organs of the body. In many of them, however, there is a period of from one to three or more days between the initial rise in the temperature and the appearance of localizing symptoms in the affected organ.

This is commonly supposed to be the prodromal stage in the inflammatory process. Often, however, with the appearance of active symptoms in the heart, lung, joint, etc., one or both middle ears show marked signs of inflammation, or spontaneously rupture. When such phenomena occur in the middle ear, it is usual to have the symptoms in other organs subside to a remarkable extent. Also the frequency with which all existing complications abate, after spontaneous rupture or free incision of the drum membrane, is significant of the powerful influence of the aural condition over other symptoms in different organs. Such being the case, may we not assume the middle ear or mastoid cells to be the point from which bacterial. invasion gains access to the circulation?

In view of the increased demands upon vital organs in these diseases, their resistance to toxic influences must of necessity be lowered. Muscle tissue is not often attacked but serous cavities and joints of cartilaginous and serous membrane structure are particularly susceptible to lowered vitality and consequent inflammation. Thus the valves of the heart, the lungs, serous cavities and joints are commonly involved. If, at such a time, the middle ear or mastoid cells send bacteria into the blood stream, the devitalized organs are in a position to absorb them, and they themselves become foci of infection to still further increase the toxemia of the patient.

This is rather a new theory, but should it prove to be correct, would be of great importance. It is too broad and complicated a sub- time.

It is the writer's intention to make this paper mainly a collection of facts found in the records of the autopsy cases and not to discuss the theories involved.

The following cases illustrate the prominent rôle played by the middle ear and mastoid cells in the contagious diseases.

CASE I. A. J., 3 weeks old. Diphtheria. Admitted to hospital Jan. 6, 1908. Was not very ill. Had no special symptoms until March 6, when she began to cry continuously. Ears examined and found normal in appearance. March 13 temperature rose to 103. March 14 edema of the scalp and chest with marked inflammation of these areas. March 17 temperature again normal. March 19 died without any special symptoms.

Autopsy.-Acute pericarditis-pleuritis-peritonitis. Both middle ears contain an acute process which consists of an elevated pink excresence. (Note on record reads.) It would appear the lesions in the ears are primary and the lesions elsewhere in the body are secondary to this.

CASE 2. I. N., 21/2 years old. Diphtheria. Child was sick for three weeks before admission to the hospital in April, 1910. She was an undeveloped, underfed baby in critical condition. Marked prostration. Nasal discharge. Slight dullness at base of right lung. Pulse rapid and weak. No vomiting. Temperature 101.6 April 8. Pulse dropped to subnormal. Heart action very weak with gallop rhythm. Failed and died. Child was too sick to make careful examination at any time.

Autopsy.-Broncho-pneumonia. Acute pericarditis. Empyema. Acute peritonitis. Both middle ears and mastoid filled with creamy, yellow pus. South Department record gives cause of death as diphtheria and empyema.

Case 3. R. W., ? years old. Diphtheria. Admitted to hospital July 27 with typical diphtheria symptoms. Temperature 101.6. Slight nasal discharge. Aug. 8 developed a gallop rhythm of the heart. Cold extremities. Some vomiting, etc. From this time on patient continued to gain and was very comfortable. Temperature practically normal. Sept. 8. Today shows marked weakness. No special symptoms. Following day died, apparently from paralysis of respiration.

Autopsy.-Slight edema and congestion of the brain. Chronic appendicitis. Chronic pleuritis. both drum membranes intact. Both middle ears contain small amount of gummy, yellowish fluid. No reason to suspect ears during life.

Case 4. A. D., 17 months old. Diphtheria. Admitted to hospital Aug. 10, 1906. For past week has been fretful. No vomiting. Marked dyspnea and prostration for 24 hours. No dullness in lungs but has moist coarse râles. Pulse rapid and weak. No evidence of laryngeal stenosis. Marked pallor and cyanosis. Gradually became comatose and died same day. Ears examined and found normal.

Autopsy.-Broncho-pneumonia. Edema and softening of brain. Chronic pachymeningitis. General lymphoid hyperplasia. Both middle ears con- 
tain purulent fluid extending into the mastoid cells on the right.

Case 5. R. C., 1 year old. Diphtheria. Admitted to hospital Dec. 10, 1909. Was not very ill. No nasal or aural discharge. Temperature 99.6. Dec. 18. Since yesterday patient has been greatly prostrated. Poor pulse and slight rise in temperature (101). Heart and lungs negative. No special symptoms of any kind, except stools show pathological conditions. Grew gradually weaker and died the next day.

Autopsy.-Edema of brain. Acute otitis and mastoid. Right middle ear contains thick, gelatinous yellow pus. In left is dark brown fluid. Same condition in mastoid. Culture, streptococcus.

Case 6. J. G., 2 years old. Scarlet fever. Admitted to hospital on April 29, 1900. Marked prostration. Temperature, 104.8. Cervical glands indurated. Heart action rapid but regular. Lungs normal except few slight scattered râles. May 7, marked septic temperature since last note. Has grown steadily worse. Heart rapid and feeble. Lungs filling up with fine and coarse râles. May 30 , condition steadily growing worse. Lungs filling up. Died. Temperature 105.

Autopsy.-Mastoiditis. Meningitis. Lungs not specified in autopsy report.

Anatomical Diagnosis.-Acute otitis media and mastoid. Acute ethmoiditis and sphenoiditis. Both middle ears and mastoid filled with pus. No aural symptoms in records.

CASE 7. L. G., 12 years old. Scarlet fever. Admitted to hospital on March 31, 1909. Marked prostration. Temperature 103. Pulse 120. Semiconscious and very restless. April 6. spontaneous rupture of right ear. April 8, much better breathing, pulse, etc. Mental condition also improved and is quite comfortable. Apr. 12, spontaneous rupture of left ear. Both now discharging. April 16, failing rapidly. Profuse discharge from both ears. April 24, swelling and edema over left mastoid. Mastoid opened. Right mastoid shows no symptom of being involved at this time. Temperature 105. April 28, temperature at 104. Conditions remain the same. May 3 , continued to yrow weaker and died.

A utopsy.-Pleuritis. Endocarditis. Nephritis. Both middle ears and both mastoids filled with pus. Both ethmoids and sphenoids also infected.

CASE 8. R. S. C., 8 years old. Scarlet fever. Admitted to hospital Nov. 6, 1905. Physical examination negative at time of entrance except scarlet fever rash. Following day became much prostrated. Temperature rose to 103 . Had marked delirium, etc. During the night both ears ruptured spontaneously. Temperature rose steadily for two days and conditions became worse. Two days later developed slight facial paralysis on right side. White count 21,600 . Right mastoid opened and no free pus found. Some rough bare bone in middle fossa.

Autopsy.-Septecemia. Acute nephritis. Acute pleuritis. Double mastoiditis. One operated on.

Case 9. M. L. C., age 12 years. Scarlet fever. Admitted to hospital Dec. 19, 1908. Patient is de- lirious. Pulse 103. Râles over both lungs and slight dullness at the base of the right lung. Dec. 26 , condition has been the same until today, when she is brighter and less delirious. Marked septic temperature still. Dec. 30, again delirious and much weaker. Patient continued with septic temperature in same condition until Jan. 7, when she died. Few hours before death showed stight facial paralysis.

Autopsy.-Acute pericarditis. Acute bronchitis. Congestion of the lung. Left middle ear filled with pus. Right middle ear and mastoid filled with pus.

Case 10. M. H., 2 months old. Diphtheria. Admitted to hospital Sept. 21, 1907. Not very ill, and continued to gain until the $29 \mathrm{th}$, when she suddenly began to fail. Some vomiting at this point. Does not take nourishment well. Sept. 30, died without any special symptoms. Temperature normal. No symptoms pointing to ears at any time.

Autopsy.-Broncho-pneumonia. Edema of the brain. Both middle ears and mastoids contain pus.

Cases 1, 3, 5 and 10 illustrate a peculiar state of affairs. All were diphtheria patients and each of them was not especially ill upon entrance to the hospital. Each improved or showed no symptoms for sixty-seven, forty-four, eight and ten days respectively. Then they developed weakness or prostration and gradually died without definite symptoms of any kind. The autopsies showed all of them to have had double acute middle ear disease; and three of the four cases, either single or double mastoiditis. Not one of the four showed any reason to suspect the middle ear during life.

Cases two and four were also diphtheria patients and were very much prostrated on admission to the hospital and died the same day. Both had severe complications in other organs, which may have been secondary to middle ear infection or mastoiditis, both of which were present in each case. In neither case was the ear suspected of being diseased during life. Elevated temperature was present, but was attributed to heart, lung or kidney inflammation, which was present and active.

Cases 6, 7, 8 and 9 were all scarlet fever patients and showed a marked contrast to the diphtheria cases. Each of them was very septic on admission; each had high temperature and severe complications other than the middle ear or mastoid. All four of these cases had mastoiditis unsuspected on either one side or both. The question may again be raised as to whether the primary infection was not in the middle ear or mastoid cells, and the inflammation of the heart, lung or kidney secondary to them. In support of this theory may be cited cases 2 and 8 , in which the records state; either free paracentesis or spontaneous rupture was followed by immediate and "marked relief from vomiting, difficult breathing and high temperature."

In cases 6 and 9 , neither middle ear or mastoid was suspected during life; both, however, 
"had pus in the mastoid cavity. Cases 7 and 8 had a mastoid operation on one side, not followed by relief of symptoms. At the autopsy, the opposite side in each case was found to be filled with pus. Neither of the unsuspected mastoids had the ordinary symptoms during life.

Granting middle ear complications to be of greater importance than we have believed them to be in the past, we face a very ugly question. Diagnosis is extremely difficult and treatment in many cases would be equally hard to carry out. A study of the clinical records of these autopsy cases prove symptoms to be practically nil in many instances. The common every-day methods of diagnosis are not at our disposal to make use of. As you have observed, most of the fatal cases mentioned occurred in very young children. All of them were under 12 years of age and many of them under two years. The sensation of pain is eliminated from the situation. Restlessness or fussiness are occasionally seen, but can never be depended upon. Swelling of the posterior wall of the canal is impossible as the young child has no bony wall. Swelling of the drum membrane and edema over the mastoid are positive signs of diagnosis only when present; as the autopsy records plainly demonstrate, pus may be present in either middle ear or mastoid, with no suspicion of these two symptoms present.

Either one of two courses is open to us. We must of necessity develop new and improved methods of diagnosis, or fail in our mission as aurists in these particular cases.

The newer methods of diagnosis are scarcely available for several reasons. It would be somewhat difficult to $x$-ray these cases on account of the ages of the patients. Such a procedure at the bedside of very sick cases, would be practically impossible. Patients with contagious diseases would never be allowed in special x-ray! rooms, and no x-ray machine I know, could be carried into a contagious hospital. The only solution of this problem would be a special $x$-ray room for the particular purpose. The $\mathrm{x}$-ray, so far as I know, is an untried experiment in such cases, and probably would be of little value, except possibly in the very acute scarlet fever and mastoid cases, wherein a large amount of pus is present. In view of the extreme necessity, however, for new methods of diagnosis, the $\mathrm{x}$-ray should be tried out.

The Mosher transillumination method would be ideal if we had adults and not very young children to deal with. The apparatus is simple, small, could be easily sterilized, and taken from one patient to another. It would further have the advantage of being used at the bed of very sick patients, without moving or otherwise disturbing them. In its present form, however, it is useless in the class of cases we most desire to use it upon, because of the age of the patient. Some modifications of this instrument may be developed, and it would be of the greatest assistance. In fact at the present time, a modi- fication of this instrument is about the only hope I see upon the horizon of these cases.

In view of the large percentage of aural involvement in very sick and fatal cases, the closest possible watch must be kept on the middle ear. Naturally such close observation will be rewarded by finding many inflamed and swollen drum membranes. Once determined, this condition is easily handled; but as distinct stereotyped symptoms are lacking, the observer must be prepared to note the merest suspicion of trouble in this locality and possess sufficient skill to note the slightest trace of altered natural conditions.

With active symptoms present in any organs of the body, particularly in the heart, kidney, pleura or joints, suspicion should be directed to the middle ear as the original focus of infection. If prominent symptoms are present in this locality, prompt and radical measures must be taken to overcome them. Should one fail to find the common symptoms of inflammation in the middle ear or mastoid cells, those organs are by no means free from suspicion on that account. It is possible, the appearance of an infected middle ear may be altered at different hours of the day and night. An apparently normal looking drum membrane in the morning, may have a different appearance in the late afternoon or evening. It would be interesting to know whether or not there was any change in the appearance of the middle ear at the time of the two extremes of a septic temperature; providing of course the fluctuation was inflamed by aural conditions.

In the treatment of such cases, there is but one course, viz. wherever pus is present, it must be thoroughly drained. This sounds simple enough to any physician reasonably familiar with aural work; but it fails to be as simple as it would first appear. To establish drainage in a comparatively healthy patient is simple enough, but to keep such drainage open in a very sick patient is an entirely different matter. Repeated incisions of the drum membrane are often called for and should be resorted to as long as necessary. Ether operations should of course be avoided as much as possible when important organs are involved; but they are never absolutely contra-indicated when necessity demands. This has been proven many times in the Boston City Hospital. Very sick patients will take ether better than would be supposed. The free drainage of pus, with its attending toxins, will do far more good, than ether with its irritation to inflamed tissue will do harm.

Ice bags should never be used in seriously ill patients in the contagious diseases. Symptoms in the middle ear or mastoid should be encouraged as much as possible. Ice bags or other methods of cold applications very effectually mask such symptoms and should be absolutely avoided. Over-conservatism will jeopardize the life of the patient. Treatment must be broad- 
minded, courageous and thorough. It must also be prompt and decisive. Hours count tremendously in this respect. Toxins must be eliminated before the system is overwhelmed to the point from which it can never recover. Unlike pneumonia, typhoid, tonsillitis, etc., this is not a self-limited process. The moment the pus is liberated, the toxins cease to be absorbed to any extent, and if the patient has sufficient vitality, the ones already in circulation will be taken care of by nature herself.

The matter of prompt and thorough treatment cannot be too strongly emphasized. The failure of present day treatment in many cases, is a lack of skillful diagnosis in the first place, and failure to promptly relieve dangerous symptoms in the second place. Practically every case of brain abscess and every infected jugular vein is the result of failure to diagnose and treat comparatively simple conditions in the early stages.

Total number of cases..........

Head not opened...............

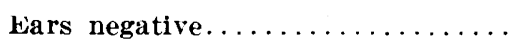

Percentage of aural involvement when conditions were noted in the records $\ldots \ldots \ldots \ldots \ldots \ldots \ldots$ AURAL COMPLications.

A.- Unilateral otitis media........

B.-Bilateral otitis media...........

C.-Unilateral mastoiditis........

D. - Bilateral mastoiditis..........

BRONCHO PNEUMONIA.

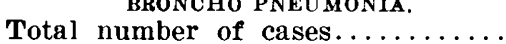

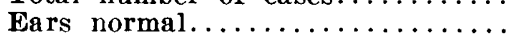

A.- Cnilateral otitis media.......

B.-Bilateral otitis media..........

C.-Unilateral mastoiditis.........

D. - Bilateral mastoiditis.........

LORAR PNEUMONIA.

Total number of cases...........

Without otitis media $\ldots \ldots \ldots \ldots$.

A.- Inilateral otitis media.......

B.-Bilateral otitis media..........

C.-Unilateral mastoiditis........

D. - Bilateral mastoiditis.........

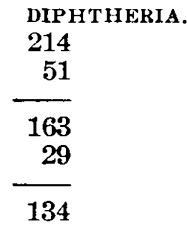

$82 \%$

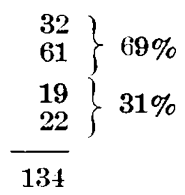

$\left.\begin{array}{r}\begin{array}{r}57 \\ 13\end{array}-23 \% \\ \hline 44-77 \% \\ 12 \\ 14 \\ 5 \\ 13\end{array}\right\} 45 \%$

$13-92 \%$

$1-8 \%$

$9\} 662-3 \%$

$\left.\begin{array}{l}1 \\ 2\end{array}\right\} 331-3 \%$
CLINICAL CASES.

SCARLET FEVER.

Total number of cases . . . . . 746

With otitis media ........... 65-11\%

Without otitis media .........681

MEASLES.

Total number of cases ........456

With otitis media ........... 125-28\%

Without otitis media $\ldots \ldots \ldots \ldots \ldots .331$

DIPHTHERIA.

Total number of cases ....... 962

With otitis media $\ldots \ldots \ldots \ldots \ldots \ldots, 28-2.9 \%$

Without otitis media ........ 934

MIXED INFECTIONS.

Total number of cases ........ 68

With otitis media............. $30-44 \%$

Without otitis media ......... 38

2232 cases in all.

333 AUTOPSIES.

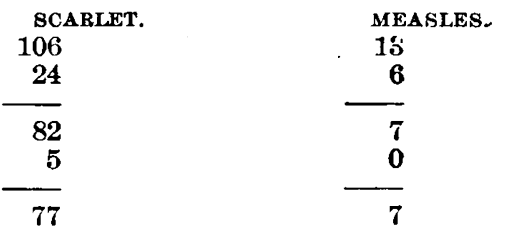

$94 \%$

$100 \%$
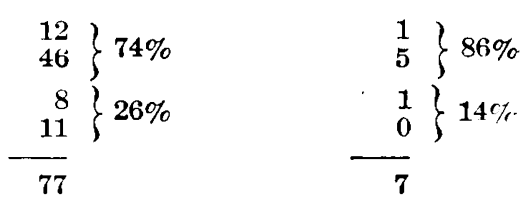

23

$\left.\begin{array}{r}-\frac{2}{21}-91 \% \\ 4 \\ 13 \\ 1 \\ 3\end{array}\right\} 26 \%$

8

$1-12.5 \%$

7-87.5\%

$2\} 100 \%$

0

o

0

0

0

0
THE DIAGNOSIS OF FEEBLE-MINDEDNESS AMONG IMMIGRANTS.

BY J. G. WILSON, M.D.

A. A. Surgeon, U. S. P. H. S., Ellis Island, New York.

The differential diagnosis of the high grade feeble-minded individual from the normal is an exceedingly difficult and complex problem.
There are all grades of human intelligence, and these grades group themselves into strains which are hereditary and can be traced with a fair degree of accuracy through successive generations. There is the strain of high intellectual ability which is easily recognized. This is fol. lowed by the middle grade or average, which is in turn to be differentiated from the feeble- 